\section{Reversal learning: The effects of conceptual and perceptual training in the absence of differential observing responses*}

\author{
HOWARD H. KENDLER† and JAMES W. WARD \\ University of California, Santa Barbara, Calif. 93106
}

Nursery school children received either verbal labeling (V), perceptual cue $(Q)$, or control (C) pretraining prior to a reversal shift problem involving sets of cues that differed in terms of brightness (B) or saturation (S). When S was relevant, differences in pretraining had no effect, but, when $B$ was relevant, the $V$ group reversed more rapidly than did the $Q$ and $C$ groups.

Explanations of discrimination-shift behavior have either emphasized perceptual (Zeaman \& House, 1963; Tighe \& Tighe, 1968) or conceptual processes (Kendler, 1971; Kendler \& Kendler, 1962, 1968). As is the case for most theoretical controversies in psychology, the dispute cannot be resolved by the results of a crucial experiment, no doubt due to the fact that one simple or single assumption is not at stake, but, instead, there is at stake a constellation of integrated premises that cannot presently be separated and evaluated independently. As a result of this state of affairs, the controversy has generated a variety of experimental data that typically have been interpreted as favoring one of the competing viewpoints, usually the one preferred by the $E$.

One such phenomenon is concerned with the relative effectiveness of perceptual orienting and symbolic representational responses on discrimination-shift behavior. A recent study (Kendler, Glasman, \& Ward, 1972 ) reports that preschool children who were trained to apply different labels to rectilinear and curvilinear lines were able to execute a reversal shift more rapidly than could those who learned to attend to the critical cues, an angle and a curve, that differentiated the two sets of lines. These perceptually trained Ss, in turn, were superior to a control group that

*This research was supported by National Science Foundation Grant GB-19323. The authors wish to acknowledge with thanks the assistance of Lynette D. Glasman, who helped collect some of the data. The authors also wish to express gratitude to the directors, teachers, and students in the following nursery schools who participated in this study: Rocking $K$ Nursery School. San Marcos Elementary School. All-Saints-by-the-Sea Nursery School, Dos Peublos Cooperative Nursery School, El Montecito Presbyterian Nursery School, and The Oaks Cooperative Nursery School.

+ Requests for reprints should be sent to Howard H. Kendler, Dept. of Psychology, University of California, Santa Barbara, Calif. 93106. had no special pretraining. The conclusion was drawn that verbal labeling had some special facilitating effect apart from any encouragement to attend to the critical features of the stimulus patterns. The beneficial effect of labeling was attributed, consistent with the developmental mediational theory (Kendler, 1971), to its tendency to generate implicit symbolic responses that served both to represent the cues of each sorting category and to control choice behavior.

The present study seeks to determine whether the findings just described can extend to a reversal-shift paradigm in which the discriminative cues are color stimuli that vary simultaneously in terms of brightness and saturation. Since these two dimensions do not require distinctive overt orienting responses, as was the case for rectilinear and curvilinear lines, the expectation would be that perceptual training, i.e., training to orient, would be less effective in the present than in the previous study (Kendler, Glasman, \& Ward, 1972). Specifically, this would mean that in the present experiment a reversal shift should be executed more rapidly by Ss who were trained to label than by those who were trained to orient perceptually to the critical features of the stimulus pattern. In addition, the previously observed superiority of perceptual training over a control group should be diminished.

\section{EXPERIMENTAL DESIGN}

The Ss were divided randomly into three groups: verbal labeling (V) Ss learned labels during pretraining to describe the stimuli of the relevant dimension, either saturation (S) or brightness (B); the cue training (Q) Ss were required during pretraining to match the stimuli of the relevant dimension, $S$ or $B$, to sample cues; the control (C) Ss received neither of these pretraining techniques. After pretraining was completed, the Ss of all groups learned a discrimination based upon differences between the stimuli of the relevant dimension, and immediately following criterion performance a reversal shift was instituted.

\section{SUBJECTS}

The $\mathrm{Ss}$ were 98 4-year-olds from six nursery schools from the Santa Barbara, California, area. Eight of the Ss were eliminated because of their failure to complete the entire experimental procedure: four Ss from Group QB were unable to learn to match the stimuli to the sample cues during pretraining; three $\mathrm{Ss}$ of the $\mathrm{CB}$ group and one $\mathbf{S}$ of the QS group failed to learn the preshift discrimination within 20 blocks of trials.

\section{STIMULI}

Munsell color chips, 3 x 5 in. in size, were used as stimuli. The response cards possessed the same hue (Red 5) but differed in $B(6 /$ or $3 /)$ and $S(/ 2$ or $/ 10$ ), resulting in a set of four response cards $(6 / 2,6 / 10,3 / 2$, and $3 / 10)$. The two cue cards to which the response cards were matched for cue training were Red $54 / 14$ and Neutral 4/ when $S$ was relevant and two gray cards, Neutral $8 /$ and Neutral 2/, when B was relevant.

\section{PROCEDURE}

Each $S$ was brought into an unoccupied room and given the Peabody Picture Vocabulary Test-Form B (Dunn, 1965). Following its administration, $S$ was told: "Now we are going to play another game with these two boxes and some cards that have different colors on them. I'm going to give you the colors one at a time. Some colors go in this box (point) and some go in this box (point). What you try to do is put the colors in the boxes they belong each time I give them to you. Now before we start putting them in the box we're going to learn something about the colors."

The $S$ then received one of the following types of pretraining: Verbal Labeling $(V)$

After $\mathrm{S}$ was shown each response card, the $E$ named it. When $B$ was relevant, light and dark were the correct labels, and when $S$ was relevant, red and brown were the appropriate names. The response cards were then shown by $E$ in blocks, with each block consisting of a random sequence of all four stimuli presented individually and successively with the $S$ instructed to name each one. A correction procedure was used, and the criterion of learning was the correct labeling of all cards for two consecutive blocks.

\section{Cue Training ( $Q$ )}

The appropriate pair of cue cards, depending on whether the relevant dimension was $S$ or $B$, were placed in front of $S$, and $E$ then matched each response card to its proper sample. 
Table 1

Mean Number of Blocks of Trials to Criterion of Learning for Pretraining, Preshift, and Reversal Learning

\begin{tabular}{|c|c|c|c|c|c|c|}
\hline \multirow{2}{*}{$\begin{array}{c}\text { Relevant } \\
\text { Dimension }\end{array}$} & \multicolumn{2}{|c|}{ Pretraining } & \multicolumn{2}{|c|}{ Preshift } & \multicolumn{2}{|c|}{ Reversal } \\
\hline & Mean & SD & Mean & SD & Mean & $\mathrm{SD}$ \\
\hline \multicolumn{7}{|l|}{ Saturation } \\
\hline V & 2.20 & .75 & 2.53 & 4.27 & 2.60 & 4.26 \\
\hline $\mathbf{Q}$ & 2.07 & .25 & 1.20 & 1.64 & 2.07 & 1.65 \\
\hline $\mathrm{C}$ & 2.07 & .25 & 5.13 & 3.98 & 3.07 & 4.22 \\
\hline \multicolumn{7}{|l|}{ Brightness } \\
\hline V & 2.87 & 1.54 & 2.00 & 3.03 & 2.80 & 4.15 \\
\hline $\mathbf{Q}$ & 4.00 & 2.94 & 4.53 & 5.43 & 5.73 & 5.58 \\
\hline $\mathrm{C}$ & 3.00 & 1.59 & 6.20 & 4.16 & 5.73 & 3.57 \\
\hline
\end{tabular}

Following this demonstration, the response cards were shown in blocks, with the $S$ instructed to point to the cue card each response card "looked like." A correction procedure was used, and criterion performance was two consecutive blocks of correct responses.

\section{Control (C)}

The E showed $S$ the response cards without any training instructions for a number of blocks approximately equivalent to the number experienced by the $S s$ in the two experimental groups, V and Q.

After pretraining was completed, discrimination training began for $S s$ in all three groups. The $S$ was required to sort each response card into one of the two boxes. Prior to each sorting response, the $S$ in both of the experimental groups was required to give the correct pretraining response, the appropriate label for $V$ Ss or the proper matching response for $\mathbf{Q}$ Ss. During discrimination training, a double criterion of correct responding was used for these Ss, a correct pretraining response and a correct sorting response. The criterion of discrimination learning for these $\mathrm{Ss}$ was two successive blocks of trials, during which all responses, both pretraining and sorting, were correct. The Ss in Group C were required only to sort correctly the response cards for two successive blocks of trials. After the criterion of discrimination learning was reached, the $S$ in the two experimental groups, $V$ and $Q$, was instructed that the pretraining response, verbal labeling or cue matching, was no longer required. The stimulus cards were removed for the $Q$ Ss. A reversal shift was irstituted, with the experimental procedure for all three groups being identical. Criterion of learning was again two successive blocks of correct responses.

Following the attainment of criterion of reversal learning, all Ss were asked.to describe which colors belonged to each sorting box. If unsuccessful, they were given the four cards and instructed to group the ones that "go together" and offer a reason why they belonged together.

\section{RESULTS}

An analysis of variance of mental age (MA) scores of the six groups of Ss revealed no significant main effects or interactions. The mean MA for all groups combined was 5 years, 5 months.

Table 1 presents the raw scores of mean blocks of trials to criterion learning for pretraining, preshift, and reversal learning. These three sets of scores were transformed into logarithms and then were subjected to an analysis of variance.

Pretraining was accomplished more rapidly when $\mathrm{S}$, as compared to $\mathrm{B}$, was relevant, $F(1,84)=10.50, \mathrm{p}<.01$. However, no significant difference occurred between verbal and cue pretraining.

A significant effect during preshift training was found as a result of pretraining conditions, $F(2,84)=9.94$, $\mathrm{p}<.01$. Planned comparisons indicated that this effect was due to the superiority of the two experimental groups, $V$ and $Q$, over the C group, $F(1,84)=19.64, p<.01$. The two experimental groups did not differ from each other, $\mathrm{F}(1,84)<1$.

An analysis of postshift training indicated that reversal learning was retarded when $B$, as compared to $S$, was the relevant dimension, $F(1,84)=$ $10.50, p<.01$. The main effect due to pretraining reached marginal significance, $\mathrm{F}(2,84)=2.84, \mathrm{p}<.10$. Further analyses were done to determine possible simple effects of the different types of pretraining on each of the two dimensions. Significant variation resulted from different pretraining procedures when $B$ was relevant, $F(1,84)=8.80$, $\mathrm{p}<.01$, but not when $\mathrm{S}$ was relevant, $\mathbf{F}(1,84)<1$. Further analyses of the significant difference for the $B$ dimension revealed that Group VB was superior to Group QB, $F(1,84)=5.21$, $p<.05$, and that the performance of Groups $\mathrm{QB}$ and $\mathrm{CB}$ did not differ, $F(1,84)<1$

The analysis of the number of perseverative errors following postshift training yielded a significant main effect due to relevant dimension, the mean for the $\mathrm{B}$ dimension being 2.67 , while the mean for the $S$ dimension was 1.53. The differences among the three groups did not achieve significance, $p>.05$.

Postexperimental questioning revealed that of the $15 \mathrm{Ss}$ in each group, 14, 10, and 4 of Groups VS, QS, and CS, respectively, could identify the two sets of stimuli with an appropriate conceptual label. Equivalent figures for the B dimension were 12,0 , and 0 . This discrepancy, 28 to 12 in favor of Ss who conceptualized $\mathrm{S}$ as compared to $\mathrm{B}$ differences, is significant, $x^{2}(1)=$ $11.5, \mathrm{p}<.01$. The difference between the two dimensions disappears if we add to the Ss who conceptualized the stimulus differences those who could correctly sort them into the appropriate two sets. The numbers of Ss who sorted the stimuli after being unable to conceptualize them were 0 , 4 , and 6 for Groups VS, QS, and CS, respectively, and 2,11 , and 8 for Groups VB, $Q B$, and $C B$. In addition, it should be noted that some $B$ Ss, 3 $\mathrm{BQ}$, and $3 \mathrm{BC}$, grouped the response cards in terms of differences in $\mathrm{S}$.

\section{DISCUSSION}

The superiority of verbal labeling over cue training in facilitating a reversal shift was not completely replicated (Kendler, Glasman, \& Ward, 1972) in the present experiment. Pretraining procedures, verbal labeling, cue training, and control had different effects, depending upon which of the two dimensions, saturation or brightness, was relevant. When the relevant dimension was saturation-the more salient of the two, as defined by the rapidity of preshift learning -reversal performance of nursery school children was unaffected by the type of pretraining. When, however, the relevant dimension was brightness, verbal labeling, consistent with previous findings (Kendler, Glasman, \& Ward, 1972 ), proved superior to cue training.

These findings, although not completely expected, can be incorporated within the developmental mediational theory (Kendler, 1971; Kendler \& Kendler, 1968). The data suggest, consistent with other evidence (Kendler, Kendler, \& Sanders, 1967; Kendler \& Watson, 1969), that the ability to categorize a set of stimuli is not only a function of the $S$ 's age, but is also influenced by the stimulus patterns. Taking this point into consideration, the evidence suggests that the $S$ s were, on the average, at different stages of conceptual development in relation to the particular values of the two dimensions. For saturation, some representational response had a high probability of occurring without any special training, as suggested by the relatively low pretraining means and 
standard deviations (see Table 1). As a result, differential results were not produced by the different pretraining procedures for the saturation dimension. In contrast, when the less salient brightness dimension was relevant, the verbal labeling encouraged representational responses that otherwise would not occur.

Further research is needed to clarify two problems raised by the above explanation. (1) The assumed equivalence of the three pretraining groups in generating an appropriate representational response when saturation was relevant is not reflected in the postexperimental interviews. This discrepancy may reflect the insensitivity of these interviews to reveal the representational processes operating during postshift performance. Perhaps more direct methods of identifying representational functioning during postshift training are needed. (2) Although the suggestion is offered that the Ss were responding at different levels of conceptual development to the two dimensions, this difference need not be considered to be sharp and distinct. One possibility is that nursery school children vary in their probability of eliciting appropriate representational responses when confronted with discriminations between cues of different relevant dimensions, with the probabilities being greater for saturation than they are for brightness. To permit a more precise analysis of experiments, such as the present one, specific probability models of representational functioning must be developed.

In spite of these limitations, the present experiment does suggest that verbal labeling can operate to facilitate reversal learning in the absence of any possible beneficial effect for perceptual orienting training. In addition, this study provides a method by which differences in overt orienting responses can be eliminated in discriminations between stimulus compounds that vary simultaneously on two dimensions.

\section{REFERENCES}

DUNN, L. D. Peabody Picture Vocabulary
Test-Form B. Nashville, Tenn: American Guidance Service, 1965.

KENDLER, $H$ H Environmental and cognitive control of behavior. American Psychologist, 1971, 26, 962-973.

KENDLER, H. H., GLASMAN, L. D., \& WARD, J. W. Verbal-labeling and cue-training in reversal-shift behavior. Journal of Experimental Child Psychology, 1972, 13, 195-209.

KENDLER, H. H., \& KENDLER, T. S. Vertical and horizontal processes in problem solving. Psychological Review, $1962,69,1-16$.

KENDLER, H. H., \& KENDLER, T. S. Mediation and conceptual behavior. In $K$. W. Spence and J. T. Spence (Eds.), The psychology of learning and motivation. Vol. 2. New York: Academic Press, 1968. Pp. 197-244.

KENDLER, H. H., KENDLER, T. S., \& SANDERS, J. Reversal and partial reversal shifts with verbal material. Journal of Verbal Learning \& Verbal Behavior, 1967, 6, 117-127.

KENDLER, H. H., \& WATSON, G. W. Economy of representation and reversal shift behavior. Journal of Verbal Learning \& Verbal Behavior, $1969,8,129-135$.

TIGHE, T. J., \& TIGHE, L. S. Differentiation theory and concept-shift behavior. Psychological Bulletin, 1968. 70, 756-761.

ZEAMAN, D., \& HOUSE, B. J. The role of attention in retardate discrimination learning. In N. R. Ellis (Ed.), Handbook of mental deficiency. New York: McGraw-Hill, 1963. Pp. 159-223. 